\title{
Ezrin-Radixin-Moesin Binding Phosphoprotein 50: A Potential Novel Biomarker in Human Papilloma Virus-Associated Head and Neck Squamous Cell Carcinomas
}

\author{
Athiva Shankar ${ }^{1}$ - Dorothy H. Crouch ${ }^{1} \cdot$ Michaelina Macluskey ${ }^{1}[$
}

Received: 6 March 2018 / Accepted: 23 May 2018 / Published online: 30 May 2018

(c) The Author(s) 2018

\begin{abstract}
High-risk human papilloma virus (HR-HPV) has increasingly been associated with head and neck squamous cell carcinoma (HNSCC), in particular oropharyngeal cancers. Ezrin-Radixin-Moesin Binding Phosphoprotein 50 (EBP50), a putative tumour suppressor, localises to the plasma membrane in suprabasal epithelium and to the cytoplasm in proliferative basal layers, and is a target for degradation by the HR-HPV E6 oncoprotein. The aim of this study was to investigate EBP50 protein expression patterns in HNSCC in a large Scottish cohort to determine if there was a correlation with HPV status and clinical outcomes. EBP50 expression patterns were assessed in 156 HNSCC including oropharyngeal (37.8\%), laryngeal (24\%), oral (19\%) and other sites (18.5\%), which were genotyped for presence of HR-HPV. HNSCC were generally negative for membranous EBP50. EBP50 expression was either cytoplasmic/absent, being 'predominantly cytoplasmic' in 76 (49\%), 'weak/negligible cytoplasmic' in 44 (28\%), 'strongly cytoplasmic' in 5 (3\%), 'heterogeneous' in $26(17 \%)$ and 'other' in 5 (3\%) samples. Forty tumours (25\%) were positive for HPV DNA, predominantly HR-HPV 16, and 44 (28\%) were p16 positive. The majority of tumours (71\%) with 'weak/negligible cytoplasmic' EBP50 expression originated in the oropharynx were more likely to have positive neck nodes, overexpression of p16 and positive tumour HR-HPV status $(P<0.001)$. Differences in EBP50 levels between oropharyngeal and non-oropharyngeal tumours may be linked to degradation of EBP50 by HR-HPV, and loss of EBP50 may therefore be a surrogate biomarker for HR-HPV infection in oropharyngeal tumours.
\end{abstract}

Keywords EBP50 $\cdot$ Head and neck squamous cell carcinoma $\cdot$ Human papilloma virus $\cdot$ Oropharyngeal cancers

\section{Introduction}

In 2014 , there were 11,400 new cases of head and neck squamous cell carcinoma (HNSCC) in the United Kingdom and over the last decade, incidence rates have increased by almost a quarter [1]. Despite improvements in treatment, almost half the affected patients will not survive 5 years and mortality rates are set to rise to seven deaths per 100,000 people by 2035 [1]. While smoking and alcohol are the biggest risk factors, high-risk human papilloma virus (HRHPV) is implicated in the increasing rates of oropharyngeal cancer [2].

Michaelina Macluskey

m.macluskey@dundee.ac.uk

1 Unit of Oral Surgery and Medicine, University of Dundee Dental School, Park Place, Dundee DD1 4HN, UK
Ezrin-Radixin-Moesin (ERM) Binding Phosphoprotein 50 (EBP50), a PDZ (postsynaptic density 95, PSD-85; discs large, Dlg; zonula occludens-1, ZO-1) domain scaffolding protein, also known as $\mathrm{Na}^{+} / \mathrm{H}^{+}$exchanger 3 regulatory factor 1 (NHERF1), is found in abundance at the plasma membranes of polarised epithelial cells where it regulates tissue architecture and cell migration. It functions as a molecular scaffold, promoting the assembly of macromolecular signalling protein complexes at the plasma membrane of epithelial cells, thereby regulating their activity. Major signalling pathways regulated by EBP50 include Phosphatidylinositol3-OH kinase (PI-3K)/AKT/Phosphatase and tensin homologue (PTEN) pathway, platelet derived growth factor receptor (PDGFR), epidermal growth factor receptor (EGFR) and Wnt/ $\beta$-catenin signalling [3].

Increasingly, while EBP50 is being reported as a potential player in cancer, its precise role is controversial [3]. Under physiological conditions, EBP50 localises to the apical membrane of polarised epithelial cells where it stabilises 
transmembrane receptors and junctional complexes [3]. In contrast, in numerous malignant tumours including breast, colorectal and hepatocellular carcinoma, aberrant EBP50 expression (either overexpressed, altered subcellular localisation or loss) has been observed [4-6]. These expression patterns led to the speculation that the function of EBP50 may be dependent on its subcellular location, whereby it acts as a tumour suppressor at the plasma membrane or acts as an oncogene when relocalised to the cytoplasm or its expression is lost [3]. Indeed, there is evidence for both, with cytoplasmic or membranous EBP50 reported to interfere with specific signalling pathways that either promote or suppress tumourigenesis respectively $[3-5,7,8]$.

EBP50 is a known target for degradation by the HR-HPV type 16 E6 oncoprotein [9], which contributes to carcinogenesis by disrupting multiple cellular pathways [10]. HPV16 is linked to cervical cancer development [11], and EBP50 has been shown to be downregulated in HPV16-positive cervical premalignant lesions and in cervical cancer-derived cell lines, concomitant with EGFR activation [12].

Oropharyngeal squamous cell carcinoma (OPSCC) is among the cancers with the fastest increasing incidence in Scotland [13] and the United States [14]. A recent United Kingdom study reported that $51.8 \%$ of these cancers were HPV-positive [15]. At other head and neck sites, however, HPV is not thought to be causative [16].

The aim of this study was to investigate EBP50 protein expression patterns protein in HNSCC in a large Scottish cohort, to determine if there was a correlation with HPV DNA status and clinical outcomes.

\section{Materials and Methods}

This retrospective study, approved by the Tayside Tissue Bank (TR000325), was conducted according to the guidelines outlined by the Research Governance Framework in Tayside, Scotland. Patients with HNSCC treated between January 2006 and December 2011 were selected from a single centre secondary care hospital. The inclusion criteria were patients over the age of 18 years diagnosed with a new primary HNSCC with adequate tissue available to allow immunohistochemistry (p16, EBP50) and PCR (HPV). The exclusion criteria included patients under 18 years of age, recurrent HNSCC, cases lost to follow up and inadequate quantity/quality of tissue for downstream processing.

The most widely used assay to detect the presence of transcriptionally active HPV in HNSCC is to determine the p16 status of tissue specimens by immunohistochemistry [17]. At this time all oropharyngeal cases had p16 staining carried out as part of the routine pathology reporting of cases in Tayside. A request was made for the tumours for the other head and neck sites to also have p16 staining. Slides stained for p16 were analysed by a single pathologist and scored as p16 positive if strong diffuse nuclear and cytoplasmic staining was observed in $\geq 70 \%$ of the tumour, consistent with the reporting protocol within Tayside [18]. Normal tonsillar tissue was used as a control.

Survival data and clinical information were recorded by reviewing case notes. Survival outcomes were assessed against p16 status, smoking and alcohol history, disease stage, HPV status and EBP50 expression. A completed database was transferred to Tayside Health Informatics Centre (HIC) for anonymisation via a secure NHS transfer system.

\section{Immunohistochemistry}

Coupes $(5 \mu \mathrm{m})$ of formalin fixed paraffin embedded (FFPE) archival tissue were de-paraffinised in xylene, and rehydrated in graded ethanol solutions $(100,90,70 \%)$. The sections were rinsed with $\mathrm{dH}_{2} \mathrm{O}$ before microwave antigen retrieval in $10 \mathrm{mM}$ citrate buffer ( $\mathrm{pH}$ 6.4) for $15 \mathrm{~min}$. Endogenous peroxidase activity was blocked with $10 \%$ hydrogen peroxide. After rinsing, staining was performed using the Vectastain Elite $\mathrm{ABC}$ kit according to the manufacturer (Vector Labs). Briefly, the slides were blocked with normal goat serum [20 min, 1\% BSA/PBS (w/v)], before incubating with primary EBP50 rabbit antibody (PA1-090, Thermo Fisher Scientific, 1:2000, 1\% BSA/PBS (w/v), $30 \mathrm{~min}$ ). After washing and incubating with biotinylated secondary antibody (1:200, 1\% BSA/PBS (w/v), $30 \mathrm{~min})$ the slides were incubated with Vectastain $\mathrm{ABC}$ reagent. After rinsing, the sections were developed in peroxidase substrate solution (5-8 $\mathrm{min}$ ), rinsed in water, counterstained with haematoxylin, dehydrated in graded ethanol solutions $(70,90,100 \%)$ and cleared in xylene. Tissue sections were mounted with DePeX mounting medium before visualising under a light microscope.

\section{Quantification of EBP50 Staining}

The protocol for scoring of EBP50 immunohistochemistry was adapted from Lv et al. [19]. Sections were analysed by two independent observers (AS and DHC) blinded to the sample identities. Additionally, $10 \%$ of the sections were scored by a third independent observer (MM). Differences in inter-observer scores ( 3 out of 156 tumours, $<2 \%$ ) were reconciled by re-reviewing sections. Five random representative fields ( 100 cells each) were viewed using $\times 40$ magnification. Membrane and cytoplasmic EBP50 expression patterns were scored separately as shown in Table 1.

\section{DNA Extraction}

Tissue section curls (3-5) $(5-10 \mu \mathrm{m})$ were used for preparation of genomic DNA using the QIAamp DNA FFPE 
Table 1 Quantification of immunohistochemical EBP50 staining Reproduced with permission from Lv et al. [19]

\author{
Membrane staining \\ Negative \\ Positive \\ Mixed \\ Cytoplasmic staining \\ Negligible/weak \\ Positive \\ Mixed
}

\begin{abstract}
No staining in $>10 \%$ of the tumour cells
Weak or moderate staining in $>10 \%$ of the tumour cells Combination of the above

Negligible/weak staining in $>10 \%$ of the tumour cells Moderate staining in $>10 \%$ of the tumour cells Combinations of the above
\end{abstract}

Five random representative fields of 100 cells each were viewed using $\times 40$ magnification, and each tumour allocated a staining pattern for EBP50 after quantification by two independent observers (AS, DHC). A third independent observer (MM) scored $10 \%$ of the samples

Tissue kit according to the manufacturer's instructions. Briefly, paraffin was removed with xylene and ethanol. After resuspension in Buffer ATL, the cell pellet was treated with Proteinase $\mathrm{K}\left(56{ }^{\circ} \mathrm{C}, 1 \mathrm{~h}\right.$, then $\left.90{ }^{\circ} \mathrm{C}, 1 \mathrm{~h}\right)$. After ethanol precipitation, DNA was collected on a QIAamp MinElute column before elution in Buffer ATE. Purified DNA samples were stored at $-20^{\circ} \mathrm{C}$.

\section{HPV Genotyping of Tumour Samples}

HPV DNA was detected by conventional PCR. Consensus primers specific to the $\beta$-globin gene and L1, E1 and E7 genes of the HPV virus were used. All $\beta$-globin-positive samples were initially subject to PCR with L1 primers. As the $\mathrm{L} 1$ open reading frame may be disrupted as a result of viral integration, L1-negative samples were also subject to PCR using E1 primers. To determine specific HPV isoforms, primers to the E7 genes of HPV16, 18, 33 and 52 were used [20-23]. Specificity was determined on template DNA from $\mathrm{SiHa}$ (HPV18) and HeLa (HPV16) cell lines, and plasmids containing HPV33 and HPV52 (gifts from Dr. G. Orth and Dr. W. Lancaster, International HPV Reference Centre, Karolinska Institutet, Stockholm).

PCR was carried out with template DNA (100 ng), forward and reverse primers $(0.4 \mu \mathrm{M})$, MyTaq DNA Polymerase $(0.5 \mu \mathrm{l})$ in $50 \mu \mathrm{l}$. Denatured DNA $\left(94{ }^{\circ} \mathrm{C}, 3 \mathrm{~min}\right)$ was subject to 35 cycles of PCR $\left(94{ }^{\circ} \mathrm{C}\right.$ for $15 \mathrm{~s}$, primer annealing between 50 and $58{ }^{\circ} \mathrm{C}$ for $15 \mathrm{~s}, 72{ }^{\circ} \mathrm{C}$ for $15 \mathrm{~s}$ ), with a final elongation step of $72{ }^{\circ} \mathrm{C}$ for $5 \mathrm{~min}$.

\section{Confirmation of HPV Genotypes by DNA Sequencing}

PCR products were purified using the QIAquick Gel Extraction Kit. Briefly, DNA fragments were excised from the agarose gel, solubilised with Buffer QG $\left(50{ }^{\circ} \mathrm{C}, 10 \mathrm{~min}\right)$ before isopropanol precipitation. DNA was collected on a QIAquick spin column before eluting with $\mathrm{dH}_{2} \mathrm{O}$. PCR products were sequenced by the Tayside Centre for Genomic Analysis, Ninewells Hospital. PCR products $(5 \mu \mathrm{l})$ were purified using a modification of the ExoSAP method by incubating with exonuclease I (1 U) and shrimp alkaline phosphatase (1 U) for $20 \mathrm{~min}$ at $37^{\circ} \mathrm{C}$ before inactivation at $80^{\circ} \mathrm{C}$ for $15 \mathrm{~min}$. Samples were sequenced bidirectionally using the ABI BigDye Terminator version 3.1 cycle sequencing kit (Applied Biosystems) and analysed on the Applied Biosystems 3730 DNA Analyser (Applied Biosystems). Sequence analysis was performed using MacVector version 12.03 (MacVector Inc., Waterbeach, Cambridge, UK).

\section{Statistical Analysis}

Data was analysed using SPSS (IBM Statistics Version 22). Data related to categorical variables was described in terms of number of patients (percentages) and as mean or median for continuous variables. Chi-squared test of independence was used to report on pairwise correlation between p16 status, HPV status and EBP50 expression on the one hand and social demographics and clinical/histopathological characteristics of the cohort on the other. Statistical significance was defined as $p<0.05$. Overall survival (OS) was defined as time (months) from diagnosis to death, end of study or date of last follow-up. Recurrence free survival (RFS) was defined as time (months) from diagnosis to locoregional recurrence. Disease free survival (DFS) was defined as time (months) from diagnosis to death due to head and neck cancer. Kaplan-Meier analysis was used to obtain 2- and 5-year survival curves. The Cox Proportional hazards model was used to estimate Hazard Ratio (HR) and 95\% Confidence Interval (CI) for prognostic significance of single and multiple variables. The Likelihood Ratio test was used to compare the relative strength of p16 and EBP50 expression as indicators of HPV infection.

\section{Results}

Given the heterogeneous nature of the cohort, we undertook immunohistochemistry of FFPE stratified squamous epithelium of tonsil tissue and normal oral mucosa to establish the baseline pattern of EBP50 staining in the various normal 
oral epithelia. Although EBP50 staining differed in the different layers of tonsil tissue (Fig. 1A (b)) and normal oral mucosa (Fig. 1B (b)), the staining pattern was consistent between the two tissue types. Whilst EBP50 was predominantly membranous in the suprabasal layers (Fig. 1A, B (c)) (stratum spinosum, stratum granulosum), no immunoreactivity was detected in the stratum corneum. Moving deeper from the suprabasal to the stratum basale, EBP50 staining changed from the membrane to a predominantly cytoplasmic location (Fig. 1A, B (d)), which correlated with proliferative basal layers. No nuclear EBP50 immunoreactivity was detected, and antibody specificity confirmed by blocking with recombinant GST-EBP50 protein (data not shown).

Having established the baseline EBP50 staining in oral tissue, a cohort of 156 samples from predominantly male patients (68\%) with a mean age of 65 years (range 30-90)
Fig. 1 Differential localisation of EBP50 in stratified squamous epithelium of $\mathbf{A}$ tonsil tissue and B normal oral mucosa. Immunohistochemical analysis of oral tissue sections stained with $a$ No primary antibody and $b$ antiEBP50 antibody (1:2000), and counterstained with haematoxylin. $c$ and $d$ are expanded sections of $(b)$ showing suprabasal (stratum spinosum, stratum granulosum) and basal layers (stratum basale), with membrane-bound and cytoplasmic EBP50 respectively. Little or no EBP50 staining was visible in the outer stratum corneum. Magnification $\times 100$
A
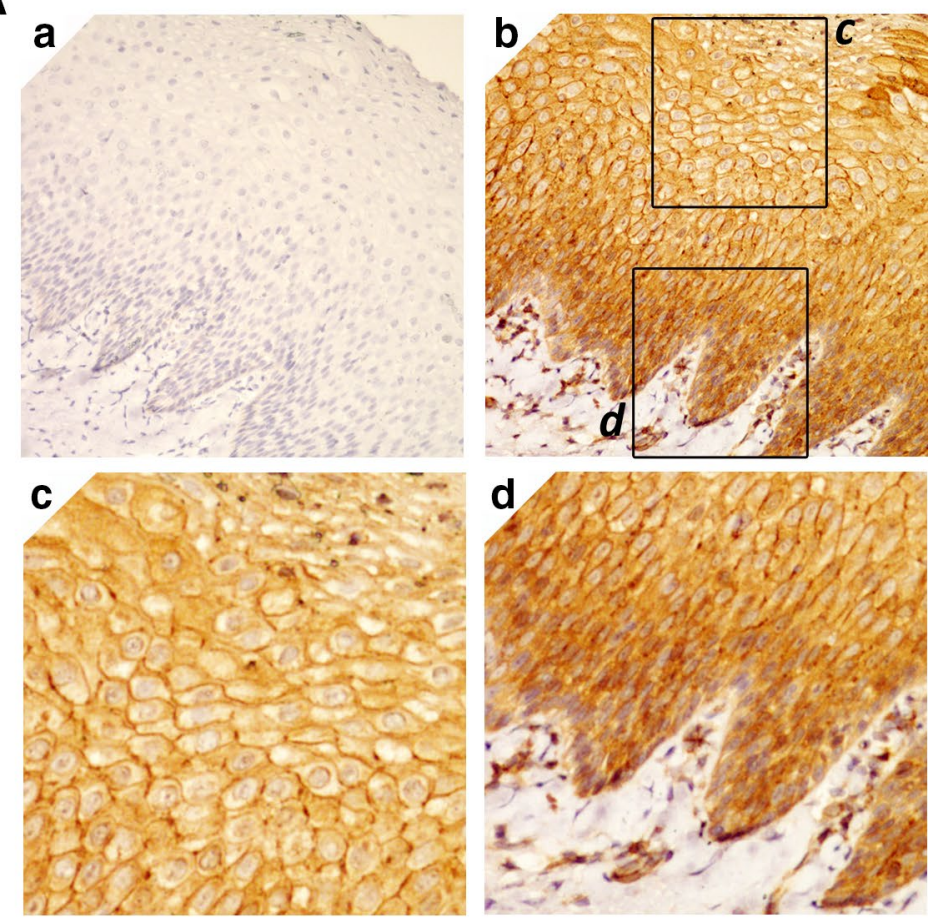

B
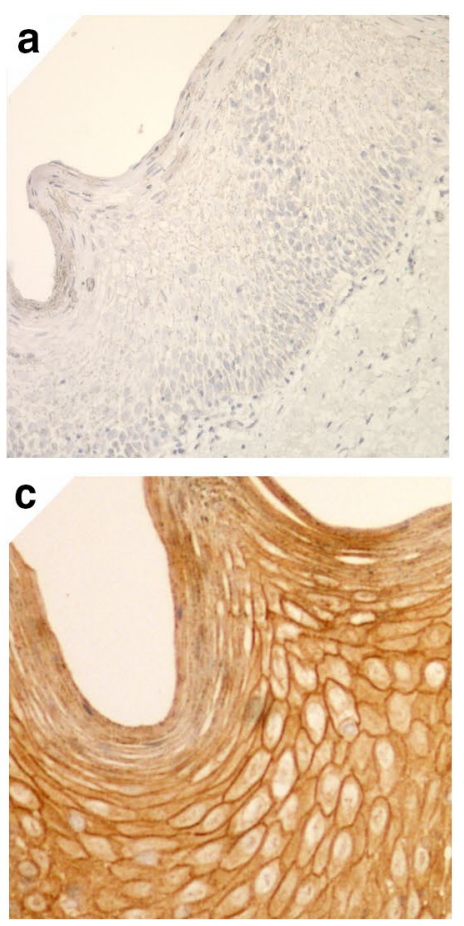
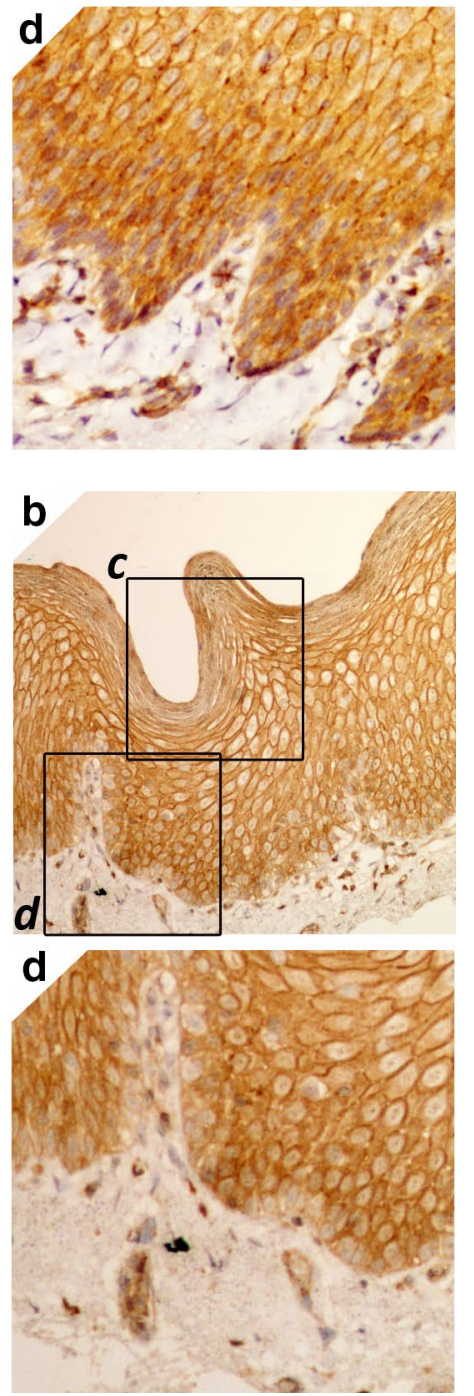
were selected. The mean length of follow up i.e. the date of diagnosis to the date of last follow up or the end of the study period of the date of death was 39 months, with $75 \%$ of patients followed up for 60 months. Treatment was surgery (19\%), radiotherapy (25\%), surgery and post-operative radiotherapy $(11 \%)$, multimodality treatment $(35 \%)$, palliation (8\%) and data missing (2\%). The tumour sites were oropharyngeal (37.8\%), laryngeal (24\%), oral (19\%) and other sites (unknown primaries, nares, nasopharyngeal) (18.5\%). Approximately half the patients presented with stage IV disease $(48 \%)$. Recurrence was observed in $16 \%$ of cases and the OS was $50.6 \%$.

EBP50 expression within the tumour cohort, quantified as described in Table 1, is summarised in Table 2, with representative immunohistochemical examples of patterns found in the majority of HNSCC shown in Fig. 2. The majority of tumours had cytoplasmic EBP50, the breakdown of which was 'predominantly cytoplasmic' in $76(49 \%)$ samples (Fig. 2b, c), 'weak/negligible cytoplasmic' in 44 (28\%) samples (Fig. 2d, e) and 'strongly cytoplasmic' in 5 (3\%) of samples. 'Heterogeneous' staining, where cytoplasmic EBP50 varied across a tumour (negative to positive), occasionally with some sparse membrane staining, was seen in 26 (17\%) samples. Five samples (3\%) had traces of membranous staining within a cytoplasmic EBP50 background. In general, with the exception of a few samples, HNSCC specimens were immunonegative for membranous EBP50 (Table 2). The lack of EBP50 expression at the plasma membrane of tumour cells (Fig. 2b, c) is consistent with the EBP50 staining in the stratum basale in normal tissue (Fig. 1). Together, these data are consistent with cytoplasmic or low levels of EBP50 being specifically associated with proliferative tissues, both normal and tumour in origin.

The clinical and pathological characteristics of the study subset were analysed based on EBP50 expression patterns (Table 3), where we found a significant association with smoking status and disease. 'Weak/negligible cytoplasmic' EBP50 expression significantly correlated with non- or ex-smokers $(p=0.019)$, late stage disease $(\mathrm{p}=0.02)$ originating in the oropharynx $(\mathrm{p}<0.001)$, positive neck nodes, overexpression of $\mathrm{p} 16$ and positive HRHPV DNA status $(p<0.001)$. In contrast, tumours with predominantly 'cytoplasmic' EBP50 expression correlated with patients with a current smoking history, were localised in the oral cavity and larynx, with no p16 overexpression and no positive nodes $(\mathrm{p}<0.001)$.

No significant associations were found between EBP50 staining patterns and grade of tumour differentiation, alcohol consumption, OS or recurrence. Patients with 'weak/ negligible cytoplasmic' EBP50 expression had the highest OS and RFS compared to those with 'predominantly cytoplasmic' EBP50 expression (OS-Median 49 months, 50 vs. $68 \%$; 5 years, 46 vs. $60 \%, P>0.05$; RFS -5 years, 64 vs. $87 \%, P=0.04$ ) (data not shown). When the impact of clinical and histological variables on RFS was investigated through univariate survival analysis, smoking status at diagnosis $(P=0.001)$, and EBP50 expression $(P=0.04)$ were found to be significantly associated with RFS.

In total, 40 samples were positive for HR-HPV DNA (25\%) and 44 were p16 positive (28\%). Of the HPV-positive samples, 39 were HPV16 positive and one was HPV 18 positive. From the anatomic breakdown, the majority of HPV-positive tumours (83\%) arose in oropharyngeal sites, whilst $17 \%$ arose in the oral cavity/other sites (Table 4). For the purposes of this study, a specimen was HPV positive if it was both p16 and HPV DNA positive. To compare p16 and EBP50 expression as indicators of HPV infection, the relative strength of their dependence with HPV DNA status was tested. Two independent Pearson's Chi Squared tests $\left(\chi^{2}\right)$ were performed to test the dependence between EBP50 expression, HPV DNA status and p16 expression and HPV DNA status (Table 5). Each test showed a strong correlation between HPV DNA status and the indicator variables. Since the $P$ values for both tests were identical $(P<0.001)$, the strength of goodness of fit of the variables was compared. The Likelihood Ratio for p16 status (87) was marginally higher than EBP50 expression (79.4).
Table 2 Summary of the immunohistochemical EBP50 staining patterns found in HNSCC in a large Scottish cohort

\begin{tabular}{lllrr}
\hline EBP50 staining & Membrane staining & Cytoplasmic staining & No. of tumours & $\%$ \\
\hline Negligible/weak & - & $-/+$ & 44 & 28 \\
Moderate & - & ++ & 76 & 49 \\
Strong & - & +++ & 5 & 3 \\
Heterogeneous & Traces $^{\mathrm{a}}$ & Variable $^{\mathrm{b}}$ & 13 & 17 \\
Heterogeneous & - & Variable $^{\mathrm{b}}$ & 13 & \\
Other & Traces $^{\mathrm{a}}$ & $-/+,++$ & 5 & 3 \\
\hline
\end{tabular}

The majority of tumours had cytoplasmic EBP50. In general, with the exception of a few minor examples, HNSCC samples were immunonegative for membranous EBP50

${ }^{\mathrm{a}}$ In a few cells within the tumour

${ }^{\mathrm{b}}$ Variation in EBP50 staining across a tumour $(-/+,++$ or +++$)$ 
Fig. 2 Cytoplasmic EBP50 is primarily found in tumour tissue. Immunohistochemical examples of sections with (a) no primary antibody control, counterstained with haematoxylin, or (b) tumours having 'predominantly cytoplasmic' or (d) 'weak-negligible' EBP50 staining. $\times 100$ magnification. Panels (c) and (e) are expanded sections from (b) and (d), showing cytoplasmic staining of EBP50, with no detectable membrane staining. Magnification $\times 400$

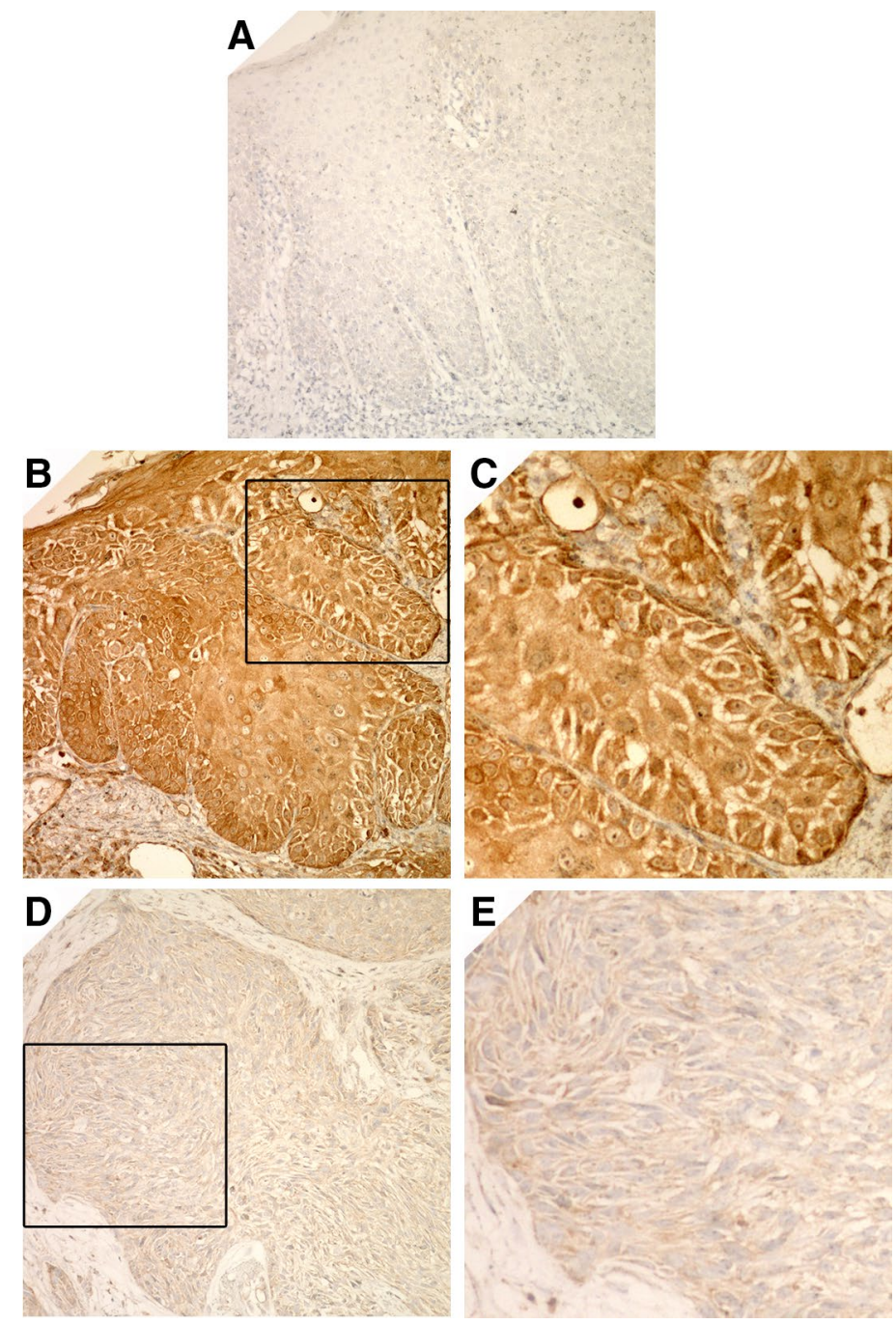

\section{Discussion}

A number of studies have reported on the potential role of EBP50 in carcinogenesis, including breast [24, 25], liver [6], colorectal [5], and gastric cancers [19]. Here, we report for the first time a study on EBP50 expression, and the impact of HR-HPV on EBP50 expression in HNSCC tumours in order to establish its validity as a novel biomarker of HR-HPV infection in HNSCC.

Collectively, our data support a model whereby the level and subcellular distribution of EBP50 may determine its function, define distinct patient profiles and identify loss of EBP50 as a potential surrogate biomarker for HR-HPV infection in oropharyngeal tumours (Fig. 3). Cytoplasmic EBP50 was found in the stratum basale in normal tissue (Fig. 3b) and in the majority of tumours (Fig. 3c), consistent with a role in this predominantly proliferative pool of epithelial cells $[4,25]$. In contrast, membrane-associated EBP50 was found in the suprabasal layers (Fig. 3a), but absent from the basal layer (Fig. 3b) and tumour sections (Fig. 3c), consistent with a role as a tumour suppressor. Contrary to others, we found no conclusive evidence for nuclear EBP50, which has been reported to regulate the $\mathrm{Wnt} / \beta$-catenin pathway [26, 27], and be a prognostic marker in breast cancer [28].

Two distinct patient profiles in this cohort were revealed when the relationship between EBP50 expression patterns and clinicopathologic characteristics was analysed, with significant associations noted with smoking history at diagnosis, site of primary tumour, disease stage, lymph node status, p16 status and HPV status (Fig. 3). The majority of tumours with 'predominantly cytoplasmic' EBP50 were more likely to be patients with a smoking history, with p16/HR-HPV-negative tumours arising in non-oropharyngeal sites such as the oral cavity and larynx with no involvement of neck nodes (Fig. 3c). In contrast, patients who were either non-smokers or ex-smokers at the time of diagnosis, had p16/HR-HPV-positive oropharyngeal tumours, presented with positive neck nodes, late stage disease, and were more likely to have 'weak/negligible 
Table 3 Social demographics and clinicopathological characteristics of patients based on EBP50 expression

\begin{tabular}{|c|c|c|c|c|c|c|}
\hline \multirow[t]{2}{*}{ Variable } & \multicolumn{6}{|c|}{ EBP50 expression patterns } \\
\hline & $\begin{array}{l}\text { Predominantly } \\
\text { cytoplasmic }\end{array}$ & $\begin{array}{l}\text { Weak/negligible } \\
\text { cytoplasmic }\end{array}$ & Hetero-geneous & Other & Total & Pearson's $\chi^{2}$ \\
\hline \multicolumn{7}{|l|}{ History of smoking } \\
\hline Non-smoker & $9(15 \%)$ & $12(32 \%)$ & $5(22 \%)$ & $1(11 \%)$ & 27 & \multirow[t]{4}{*}{$P=0.019$} \\
\hline Smoker & $36(60 \%)$ & $8(22 \%)$ & $11(48 \%)$ & $6(67 \%)$ & 61 & \\
\hline Ex-smoker & $15(25 \%)$ & $17(46 \%)$ & $7(30 \%)$ & $2(22 \%)$ & 41 & \\
\hline Total & 60 & 37 & 23 & 9 & $129^{\mathrm{a}}$ & \\
\hline \multicolumn{7}{|l|}{ History of alcohol consumption } \\
\hline Light-moderate drinker & $33(43 \%)$ & $27(61 \%)$ & $12(46 \%)$ & $3(30 \%)$ & 75 & \multirow[t]{6}{*}{$P=0.14(\mathrm{NS})$} \\
\hline Heavy drinker & $15(20 \%)$ & $7(16 \%)$ & $8(31 \%)$ & $5(50 \%)$ & 35 & \\
\hline Non-drinker & $8(10 \%)$ & $2(5 \%)$ & $3(12 \%)$ & $0(0 \%)$ & 13 & \\
\hline Ex-heavy drinker & $2(3 \%)$ & $0(0 \%)$ & $0(0 \%)$ & $1(10 \%)$ & 3 & \\
\hline Alcohol status unknown & $18(24 \%)$ & $8(18 \%)$ & $3(11 \%)$ & $1(10 \%)$ & 30 & \\
\hline Total & 76 & 44 & 26 & 10 & 156 & \\
\hline \multicolumn{7}{|l|}{ Site of primary tumour } \\
\hline Oral cavity & $17(22.4 \%)$ & $5(11 \%)$ & $7(27 \%)$ & $1(10 \%)$ & 30 & \multirow[t]{5}{*}{$P<0.001$} \\
\hline Oropharynx & $17(22.4 \%)$ & $31(71 \%)$ & $9(35 \%)$ & $2(20 \%)$ & 59 & \\
\hline Larynx & $23(30 \%)$ & $2(4 \%)$ & $7(27 \%)$ & $6(60 \%)$ & 38 & \\
\hline Other & $19(25 \%)$ & $6(14 \%)$ & $3(11 \%)$ & $1(10 \%)$ & 29 & \\
\hline Total & 76 & 44 & 26 & 10 & 156 & \\
\hline \multicolumn{7}{|l|}{ Grade of differentiation } \\
\hline Well differentiated & $1(1 \%)$ & $0(0 \%)$ & $0(0 \%)$ & $0(0 \%)$ & 1 & \multirow[t]{5}{*}{$P=0.243(\mathrm{NS})$} \\
\hline Moderately differentiated & $32(44 \%)$ & $7(18 \%)$ & $11(44 \%)$ & $2(25 \%)$ & 52 & \\
\hline Poorly differentiated & $33(45 \%)$ & $26(67 \%)$ & $12(48 \%)$ & $6(75 \%)$ & 77 & \\
\hline Moderate-poorly differentiated & $7(10 \%)$ & $6(15 \%)$ & $2(8 \%)$ & $0(0 \%)$ & 15 & \\
\hline Total & 73 & 39 & 25 & 8 & $145^{\mathrm{a}}$ & \\
\hline \multicolumn{7}{|l|}{ Disease stage } \\
\hline Stage I & $13(18 \%)$ & $3(7 \%)$ & $4(18 \%)$ & $3(30 \%)$ & 23 & \multirow[t]{6}{*}{$P=0.02$} \\
\hline Stage II & $12(17 \%)$ & $1(2 \%)$ & $3(13 \%)$ & $2(20 \%)$ & 18 & \\
\hline Stage III & $12(17 \%)$ & $4(9 \%)$ & $6(26)$ & $3(30 \%)$ & 25 & \\
\hline Stage IVA \& IVB & $33(45 \%)$ & $32(73 \%)$ & $9(39 \%)$ & $1(10 \%)$ & 75 & \\
\hline Unknown & $2(3 \%)$ & $4(9 \%)$ & $1(4 \%)$ & $1(10 \%)$ & 8 & \\
\hline Total & 72 & 44 & 23 & 10 & $149^{\mathrm{a}}$ & \\
\hline \multicolumn{7}{|l|}{ Neck node status } \\
\hline Negative & $43(59 \%)$ & $5(11 \%)$ & $14(54 \%)$ & $8(80 \%)$ & 70 & \multirow[t]{3}{*}{$P<0.001$} \\
\hline Positive & $30(41 \%)$ & $39(89 \%)$ & $12(46 \%)$ & $2(20 \%)$ & 83 & \\
\hline Total & 73 & 44 & 26 & 10 & $153^{\mathrm{a}}$ & \\
\hline \multicolumn{7}{|l|}{ p16 status } \\
\hline Positive & $7(9 \%)$ & $32(73 \%)$ & $3(11 \%)$ & $2(20 \%)$ & 44 & \multirow[t]{3}{*}{$P<0.001$} \\
\hline Negative & $69(91 \%)$ & $12(27 \%)$ & $23(89 \%)$ & $8(80 \%)$ & 112 & \\
\hline Total & 76 & 44 & 26 & 10 & 156 & \\
\hline \multicolumn{7}{|l|}{ HPV status } \\
\hline Positive & $6(9 \%)$ & $29(83 \%)$ & $3(12 \%)$ & $1(11 \%)$ & $39^{\mathrm{c}}$ & \multirow[t]{3}{*}{$P<0.001$} \\
\hline Negative & $63(91 \%)$ & $6(17 \%)$ & $22(88 \%)$ & $8(89 \%)$ & 99 & \\
\hline Total & 69 & 35 & 25 & 9 & $138^{\mathrm{b}}$ & \\
\hline \multicolumn{7}{|l|}{ OS } \\
\hline Alive & $31(41 \%)$ & $27(61 \%)$ & $15(58 \%)$ & $6(60 \%)$ & 79 & \multirow[t]{3}{*}{$P=0.12(\mathrm{NS})$} \\
\hline Dead & $45(59 \%)$ & $17(39 \%)$ & $11(42 \%)$ & $4(40 \%)$ & 77 & \\
\hline Total & 76 & 44 & 26 & 10 & 156 & \\
\hline
\end{tabular}


Table 3 (continued)

\begin{tabular}{|c|c|c|c|c|c|c|}
\hline \multirow[t]{2}{*}{ Variable } & \multicolumn{6}{|c|}{ EBP50 expression patterns } \\
\hline & $\begin{array}{l}\text { Predominantly } \\
\text { cytoplasmic }\end{array}$ & $\begin{array}{l}\text { Weak/negligible } \\
\text { cytoplasmic }\end{array}$ & Hetero-geneous & Other & Total & Pearson's $\chi^{2}$ \\
\hline \multicolumn{7}{|c|}{ Recurrence } \\
\hline Yes & $18(24 \%)$ & $5(11 \%)$ & $1(4 \%)$ & $1(10 \%)$ & 25 & $P=0.067(\mathrm{NS})$ \\
\hline No & $58(76 \%)$ & $39(89 \%)$ & $25(96 \%)$ & $9(90 \%)$ & 131 & \\
\hline Total & 76 & 44 & 26 & 10 & 156 & \\
\hline
\end{tabular}

Significant associations were noted between EBP50 expression and smoking history, site of primary tumour, stage of disease, metastasis to cervical lymph nodes, tumour p16 and HPV status

${ }^{\text {a }}$ Patients with missing or unavailable data were excluded from analysis

bPatients with 'Equivocal' HPV status were excluded from analysis

${ }^{c}$ EBP50 staining could not be determined in one HPV-positive specimen

Table 4 Summary of HPV-positive tumours by anatomic location in the head and neck

\begin{tabular}{llcl}
\hline $\begin{array}{l}\text { Head and neck } \\
\text { subsite group }\end{array}$ & $\begin{array}{l}\text { Clinical anatomic } \\
\text { location }\end{array}$ & No. of patients & $\begin{array}{l}\text { Type of } \\
\text { high-risk } \\
\text { HPV }\end{array}$ \\
\hline Oral cavity & Dorsum of tongue & 1 & HPV 16 \\
& Palate & 1 & HPV 16 \\
& Anterior 1/3 of tongue & 1 & HPV 16 \\
Oropharynx & Base of tongue & 9 & HPV 16 \\
& Tonsil & 24 & HPV 16 \\
Other & Unknown primary & 1 & HPV 16 \\
& Pharynx & 1 & HPV 16 \\
& Nares & 1 & HPV 16 \\
& Nasal cavity & 1 & HPV 18 \\
\hline
\end{tabular}

The majority of HPV-positive tumours (33/40; 83\%) arose from the oropharynx with the tonsils and base of the tongue being the most common subsites of origin. An overwhelming majority of the HPVpositive tumours were infected with high-risk type 16

cytoplasmic' EBP50 expression (Fig. 3d), suggesting these may have a different pathology from those expressing 'predominantly cytoplasmic' EBP50. The improved survival in this cohort is in keeping with other published work in this area suggesting that although these patients have aggressive disease they respond well to treatment [13].
HR-HPV E6 oncoprotein has the potential to interact with and mediate the degradation of a subset of PDZ-containing cell polarity regulators including human Dlg $[29,30]$ and EBP50 [9], and specific subcellular pools of cell polarity proteins [31, 32]. Consequently, reduced EBP50 expression in the group of patients with oropharyngeal tumours may be a result of HR-HPV E6-mediated degradation of membrane pools of EBP50 (Fig. 3d), thereby disrupting cell polarity which is central to the control of cell proliferation and cell survival [10].

The relationship between EBP50 expression as an independent variable and clinical outcomes of patients in the cohort showed that patients with 'predominantly cytoplasmic' EBP50 expression had the worst prognosis whilst reduced cytoplasmic EBP50 expression was associated with better OS and RFS. Previous reports on clinical outcomes and EBP50 expression are varied, seemingly linked to subcellular localisation of the protein [3]. In invasive breast carcinomas, cytoplasmic EBP50, high or low, did not correlate with OS or DFS [28]. Likewise, in gastric cancer no correlation was found between OS rates and EBP50 expression [19]. In our cohort, a marked improvement was observed in the DFS survival rates for the patients with 'weak/negligible cytoplasmic' EBP50expressing tumours, while the survival rate of patients with 'predominantly cytoplasmic' EBP50 steadily declined over

Table 5 EBP50 expression and p16 status correlation with HPV DNA status using Pearson's $\chi^{2}$ tests

\begin{tabular}{|c|c|c|c|c|c|c|}
\hline \multirow{2}{*}{$\begin{array}{l}\text { HPV DNA status } \\
(\mathrm{n}=156)\end{array}$} & \multicolumn{2}{|c|}{ p16 Overexpression } & \multirow{2}{*}{$\begin{array}{l}P \text { value and likeli- } \\
\text { hood ratio value }\end{array}$} & \multicolumn{2}{|l|}{ EBP50 expression } & \multirow[t]{2}{*}{$P$ value and likelihood ratio value } \\
\hline & Positive & Negative & & Weak/negligible & Other & \\
\hline Positive & $39(75 \%)$ & $13(25 \%)$ & \multirow{2}{*}{$\begin{array}{l}P<0.001 \\
87\end{array}$} & $38(73 \%)$ & $14(9 \%)$ & \multirow{2}{*}{$\begin{array}{l}P<0.001 \\
79.4\end{array}$} \\
\hline Negative & $5(5 \%)$ & $99(95 \%)$ & & $6(6 \%)$ & $98(63 \%)$ & \\
\hline
\end{tabular}

Two independent Pearson's $\chi^{2}$ tests showed strong correlation between HPV DNA status and both p16 overexpression and EBP50 expression pattern 
Fig. 3 Subcellular localisation and levels of EBP50 define a distinct patient profile in HNSCC and may determine its function. In normal epithelium, EBP50 is present on the membrane (thick black line) in suprabasal layers (S. spinosum, S. granulosum) ( $a$ tumour suppressor function), whilst in the basal layers (light shading), it is present in the cytoplasm ( $b$ proliferative function). In diseased tissue, high levels of cytoplasmic EBP50 (darker shading) are predominantly found in nonoropharyngeal HNSCC, whilst HR-HPV associated tumours, which are predominantly found in the oropharynx, have little or no EBP50

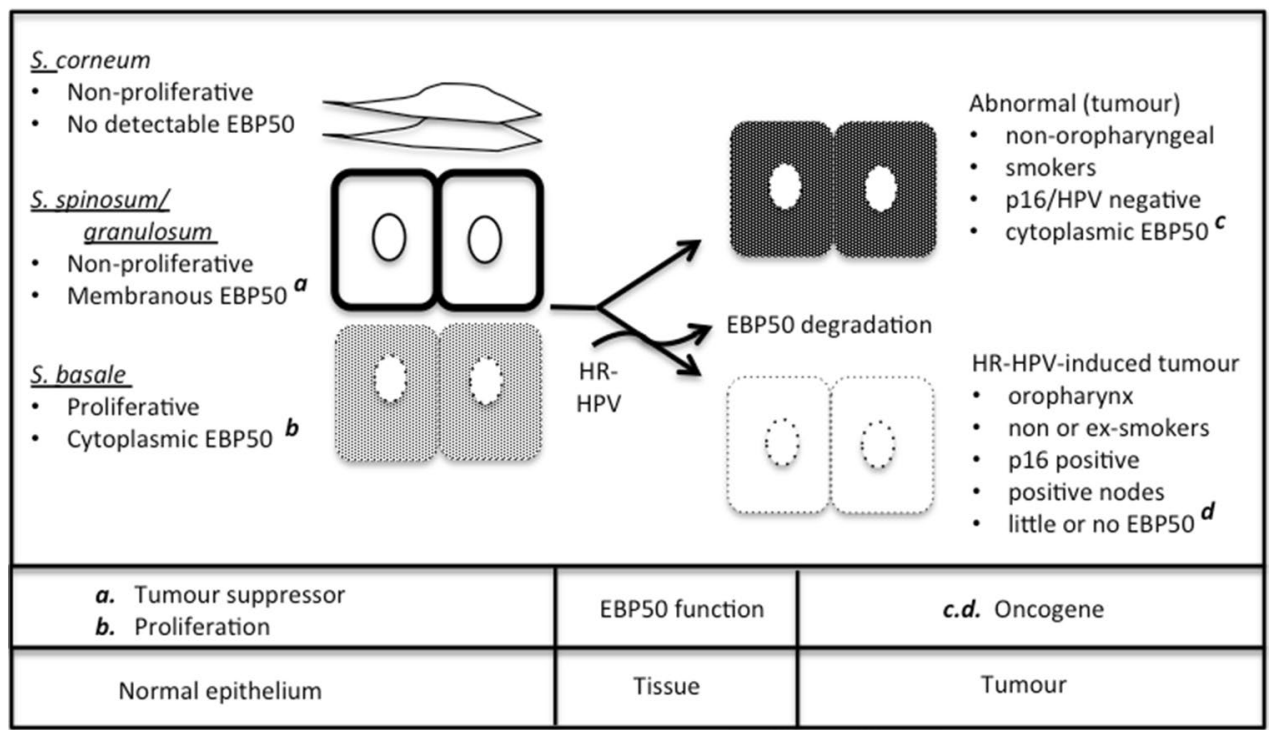

time. Since DFS analysis was not statistically significant, EBP50 expression may not be a valuable indicator of prognosis in patients with HNSCC.

The molecular mechanisms, which are known to regulate the subcellular localisation of EBP50 and as such its function, are varied, ranging from phosphorylation or interaction with ERM proteins [3]. Functionally, membrane-associated EBP50 has been shown to regulate the adherens junction proteins E-cadherin and $\beta$-catenin at the cell surface [8]. In addition, EBP50 regulates PTEN, a negative regulator of PI-3K/AKT signalling at both the membrane and in the cytoplasm [3, 7]. Although we cannot attribute a function with the redistribution of EBP50 to the cytoplasm in our cohort, collectively, our data are consistent with a tumour suppressor function at the cell membrane, and oncogenic function in the cytoplasm (Fig. 3), possibly by regulating the $\mathrm{PI}-3 \mathrm{~K} / \mathrm{AKT} / \mathrm{PTEN}$ axis in the cytoplasm and $\mathrm{Wnt} / \beta$-catenin signalling through the adherens junction proteins E-cadherin and $\beta$-catenin at the cell membrane $[3,7,8]$.

\section{Conclusion}

In the majority of oral cancers, EBP50 expression was predominantly cytoplasmic. A smaller group of tumours, the majority involving the oropharynx, demonstrated reduced/ negligible cytoplasmic EBP50 expression, which strongly correlated with a positive HR-HPV status. These data suggest that EBP50 may act as a tumour suppressor when localised at the plasma membrane or act as an oncogene when relocalised to the cytoplasm or its expression is lost. In addition, since EBP50 expression differs between oropharyngeal and non-oropharyngeal tumours, this may be linked to its degradation by HR-HPV and as such, could serve as a surrogate marker for HR-HPV.

Acknowledgements The authors would like to thank Dr. Sharon White for help with the p16 scoring of specimens, Dr. Margaret Florence for technical assistance, and Dr. Alison Harrow for advice.

Funding This studentship (AS) was funded by the Tattersal Fund (Grant Code 290.080355).

\section{Compliance with Ethical Standards}

Conflict of interest The authors declare that they have no conflict of interest.

Ethical Approval All procedures performed in studies involving human participants were in accordance with the ethical standards of the Tayside Tissue Bank (TR000325), was conducted according to the guidelines outlined by the Research Governance Framework in Tayside, Scotland and with the 1964 Helsinki declaration and its later amendments or comparable ethical standards.

Informed Consent For this type of study, formal consent was not required.

Open Access This article is distributed under the terms of the Creative Commons Attribution 4.0 International License (http://creativeco mmons.org/licenses/by/4.0/), which permits unrestricted use, distribution, and reproduction in any medium, provided you give appropriate credit to the original author(s) and the source, provide a link to the Creative Commons license, and indicate if changes were made. 


\section{References}

1. Cancer Research UK. 2018. http://cancerresearchuk.org/healt h-professional/cancer-statistics/statistics-by-cancer-type/oralcancer. Accessed 22 Jan 2018.

2. Taberna M, Mena M, Pavón MA, Alemany L, Gillison ML, Mesía R. Human papillomavirus related oropharyngeal cancer. Ann Oncol. 2017;28:2386-98.

3. Vaquero J, Nguyen Ho-Boldoires CA, Fouassier L. Role of the PDZ-scaffold protein NHERF1/EBP50 in cancer biology: from signalling regulation to clinical relevance. Oncogene. 2017;36:3067-79.

4. Stemmer-Rachamimov AO, Wiederhold T, Nielsen GP, James M, Pinney-Michalowski D, Roy JE, et al. NHE-RF, a merlininteracting protein, is primarily expressed in luminal epithelia, proliferative endometrium, and estrogen receptor-positive breast carcinomas. Am J Pathol. 2001;158:57-62.

5. Hayashi Y, Molina JR, Hamilton SR, Georgescu M-M. NHERF1/ EBP50 is a new marker in colorectal cancer. Neoplasia. 2010;12:1013-22.

6. Shibata T, Chuma M, Kokubu A, Sakamoto M, Hirohashi S. EBP50, a $\beta$-Catenin-associating protein, enhances Wnt signaling and is over-expressed in hepatocellular carcinoma. Hepatology 2003;38:178-86

7. Molina JR, Morales FC, Hayashi Y, Aldape KD, Georgescu M-M. Loss of PTEN binding adapter protein NHERF1 from plasma membrane in Glioblastoma contributes to PTEN inactivation. Cancer Res 2010;70:6697-703.

8. Kreimann EL, Morales FC, de Orbeta-Cruz J, Takahashi Y, Adams H, Liu T-J, et al. Cortical stabilization of $\beta$-Catenin contributes to NHERF1/EBP50 tumor suppressor function. Oncogene. 2007;6:5290-99.

9. Accardi R, Rubino R, Scalise M, Gheit T, Shahzad N, Thomas M, et al. E6 and E7 from human papillomavirus type 16 cooperate to target the PDZ protein $\mathrm{Na} / \mathrm{H}$ exchange regulatory factor 1 . J Virol. 2011;85:8208-16.

10. James CD, Roberts S. Viral interactions with PDZ domain-containing proteins-an oncogenic trait? Pathogenes. 2016;5:8.

11. Zur Hausen H. Papillomaviruses and cancer: From basic studies to clinical application. Nat Rev Cancer. 2002;2:42-50.

12. Peng Z, Wang Q, Zhang Y, He J, Zheng J. EBP50 interacts with EGFR and regulates EGFR signalling to affect the prognosis of cervical cancer patients. Int J Oncol. 2016;49:1737-45.

13. Junor EJ, Kerr GR, Brewster DH. Oropharyngeal cancer. Fastest increasing cancer in Scotland, especially in men. Br Med J. 2010;340:c2512

14. Chaturvedi AK, Engels EA, Pfeiffer RM, et al. Human papillomavirus and rising oropharyngeal cancer incidence in the United States. J Clin Oncol. 2011;29:4294-301.

15. Schache AG, Powell NG, Cuschieri KS, et al. HPV-Related oropharynx cancer in the United Kingdom: an evolution in the understanding of disease etiology. Cancer Res. 2016;76:6598-606.

16. Javadi P, Sharma A' Zahnd WE, Jenkins WD. Evolving disparities in the epidemiology of oral cavity and oropharyngeal cancers. Cancer Causes Control. 2017;28:635-45.

17. Lewis JS Jr. p16 immunohistochemistry as a standalone test for risk stratification in oropharyngeal squamous cell carcinoma. Head Neck Pathol. 2012;6:75-82.
18. Begum S, Gillison ML, Ansari-Lari MA, Shah K, Westra WH. Detection of human papillomavirus in cervical lymph nodes: a highly effective strategy for localizing site of tumor origin. Clin Cancer Res. 2003;9:6469-75.

19. Lv X-G, Lei X-F, Ji MY, Guo X-F, Wang J, Dong W-G. Clinical significance of EBP50 overexpression assessed by quantum dot analysis in gastric cancer. Oncol Lett. 2013;5:1844-48.

20. Resnick RM, Cornelissen MT, Wright DK, Eichinger GH, Fox HS, ter Schegget J, et al. Detection and typing of Human Papillomavirus in archival cervical cancer specimens by DNA amplification with consensus primers. J Natl Cancer Inst. 1990;82:1477-84.

21. Husnjak K, Grce M, Magdić L, Pavelić K. Comparison of five different polymerase chain reaction methods for detection of Human Papillomavirus in cervical cell specimens. J Virol Methods 2000;88:125-34.

22. Tieben LM, ter Schegget J, Minnaar R, Bavinck JNB, Berkhout RJ, Vermeer BJ, et al. Detection of cutaneous and genital HPV types in clinical samples by PCR using consensus primers. J Virol Methods. 1993;42:265-79.

23. Walboomers JM, Jacobs MV, Manos MM, Bosch FX, Kummer JA, Shah KV, et al. Human Papillomavirus is a necessary cause of invasive cervical cancer worldwide. J Pathol. 1999;189:12-9.

24. Cardone RA, Bellizzi A, Busco G, Weinman EJ, Dell'Aquila ME, Casavola V, et al. The NHERF11 PDZ2 domain regulates PKARhoA-p38-mediated Nhe1 activation and invasion in breast tumor cells. Mol Biol Cell. 2007;18:1768-80.

25. Mangia A, Chiriatti A, Bellizzi A, Malfettone A, Stea B, Zito FA, et al. Biological role of NHERF1 protein expression in breast cancer. Histopathology. 2009;55:600-08.

26. Malfettone A, Silvestris N, Paradiso A, Mattioli E, Simone G, Mangia A. Overexpression of nuclear NHERF1 in advanced colorectal cancer: association with hypoxic microenvironment and tumor invasive phenotype. Exp Mol Pathol. 2012;92:296-303.

27. Lin Y-Y, Hsu Y-H, Huang H-Y, et al. Aberrant nuclear localization of EBP50 promotes colorectal carcinogenesis in xenotransplanted mice by modulating TCF- 1 and $\beta$-catenin interactions. J Clin Investig. 2012;122:1881-94.

28. Paradiso A, Scarpi E, Malfettone A, Addati T, Giotta F, Simone $\mathrm{G}$, et al. Nuclear NHERF1 expression as a prognostic marker in breast cancer. Cell Death Dis. 2013;4:e904.

29. Gardiol D, Kühne C, Glaunsinger B, Lee SS, Javier R, Banks L. Oncogenic Human Papillomavirus E6 proteins target the discs large tumour suppressor for proteasome-mediated degradation. Oncogene. 1999;18:5487-96.

30. Nakagawa S, Huibregtse JM. Human scribble (Vartul) is targeted for ubiquitin-mediated degradation by the high-risk papillomavirus E6 proteins and the E6AP ubiquitin-protein ligase. Mol Cell Biol. 2000;20:8244-53.

31. Massimi P, Gammoh N, Thomas M, Banks L. HPV E6 specifically targets different cellular pools of its PDZ domain-containing tumour suppressor substrates for proteasome-mediated degradation. Oncogene. 2004;23:8033-39.

32. Kranjec C, Banks L. A systematic analysis of Human Papillomavirus (HPV) E6 PDZ substrates identifies Magi-1 as a major target of HPV Type 16 (HPV-16) and HPV-18 whose loss accompanies disruption of tight junctions. J Virol. 2011;85:1757-64. 